\title{
Irreversible electroporation in patients with liver tumours: treated-area patterns with contrast-enhanced ultrasound
}

\author{
Linyu Zhou', Shanyu Yin ${ }^{1}$, Weilu Chai ${ }^{1}$, Qiyu Zhao², Guo Tian ${ }^{3}$ Danxia Xu and Tian'an Jiang ${ }^{1,2^{*}}$
}

\begin{abstract}
Background: Familiarity with post-IRE imaging interpretation is of considerable importance in determining ablation success and detecting recurrence. CEUS can be used to assess the tumour response and characteristics of the ablation zone. It is of clinical interest to describe the ultrasonographic findings of liver tumours after irreversible electroporation (IRE) percutaneous ablation.

Methods: A prospective study of 24 cases of malignant liver tumours (22 cases of primary liver tumours and 2 cases of liver metastases) treated by IRE ablation was conducted. Two inspectors evaluated the ablation zone in a consensus reading performed immediately, 1 day, and 1 month after IRE ablation. The gold standard method, magnetic resonance imaging $(\mathrm{MRI})$, was used to evaluate the effectiveness of the treatment at 1 month.

Results: Immediately after IRE ablation and up to 1 month later, the ablation zones gradually changed from hypoechogenicity to hyper-echogenicity on conventional ultrasound and showed non-enhancement on contrastenhanced ultrasound (CEUS). One month after IRE ablation, CEUS and MRI results were highly consistent $(K=0.78$, $p<0.05)$.

Conclusions: We conclude that CEUS may be an effective tool for assessing post-IRE ablation changes after 1 month. CEUS enables the depiction of tumour vascularity in real time and serves as an easy, repeatable method.
\end{abstract}

Keywords: Irreversible electroporation, Liver tumours, Contrast-enhanced ultrasound, Ablation, Ultrasonography

\section{Introduction}

Liver cancer is the fourth leading cause of cancer-related deaths in the world [1]. Most patients are not eligible for radical surgical resection at the time of diagnosis. Radiofrequency ablation (RFA), microwave ablation (MWA) or cryoablation is potentially curative in select patients.

\footnotetext{
* Correspondence: tiananjiang@zju.edu.cn

The study was conducted at the Department of Hepatobiliary and Pancreatic Surgery, The First Affiliated Hospital, College of Medicine, Zhejiang University 'Department of Ultrasonography, The First Affiliated Hospital, College of Medicine, Zhejiang University, No. 79 Qingchun Road, Hangzhou 310003, Zhejiang Province, P.R. China

${ }^{2}$ Department of Hepatobiliary and Pancreatic Surgery, The First Affiliated Hospital, College of Medicine, Zhejiang University, No. 79 Qingchun Road, Hangzhou 310003, Zhejiang Province, P.R. China

Full list of author information is available at the end of the article
}

However, the efficacy of ablation is limited by the size, number and location of the lesion. As a result, the effectiveness and safety of these techniques are limited for lesions adjacent to important structures, such as the bile duct, portal vein, and gastrointestinal tract. For patients with such lesions, irreversible electroporation (IRE) is considered an alternative treatment. Compared to other local ablation techniques, IRE is a promising technique. IRE is a non-thermal ablation method that induces tumour necrosis by inducing apoptosis and cell death. IRE treatment generates electric pulses that alter the cell membrane's electrical potential, leading to small nanopores and contributing to apoptosis [2]. The efficacy of IRE is unaffected by the so-called heat-sink effect. Thus, 
IRE is currently being applied experimentally and clinically in a wide range of tissues [3, 4].

Contrast-enhanced ultrasound (CEUS) allows continuous real-time observation of lesion tissue enhancement in arteries, portal veins, and advanced stages. Thus, CEUS can dynamically assess blood flow and tissue perfusion. The feasibility of using CEUS to assess ablation areas has been reported in some preliminary animal and clinical studies $[5,6]$.

CEUS can be used to assess the tumour response and the characteristics of the ablation zone. Only limited data are available on the CEUS imaging characteristics of the ablation zones after IRE ablation in humans. Previous studies on lesions after IRE have focused on the imaging manifestations of CT and MRI [7-9], though there are few studies on the performance of lesions after IRE in CEUS. For example, Lin et al. and Rennert et al. used animal experiments to explore the changes in lesions within $2 \mathrm{~h}$ and $24 \mathrm{~h}$ after IRE, respectively $[10,11]$ and the clinical case study of Niessen et al. focused on changes of lesions from 6 weeks to 1 year after IRE [12]. To understand the dynamic changes of lesions on CEUS from a few hours to 1 month after IRE, we designed and conducted this study to provide a basis for postoperative imaging follow-up. After IRE ablation, cell death occurs with electroporation of the cell membrane and changes in histomorphology. Thus, the assessment is different from those for other ablation methods. Familiarity with postIRE imaging interpretation is of considerable importance in determining ablation success and detecting recurrence.

The present study focuses on specific imaging characteristics of hepatic tumours obtained by CEUS with an intravenous contrast agent immediately, 1 day, and 1 month after US-guided percutaneous IRE ablation. Magnetic resonance imaging (MRI) was performed 1 month after ablation to investigate the therapeutic efficacy.

\section{Materials and methods}

This study was conducted with the approval of our institutional review board. The research conformed to the Declaration of Helsinki. Before receiving CEUS treatment, patients signed an informed consent form for imaging data analysis.

\section{Patients}

We conducted a single-centre prospective study to evaluate and describe the CEUS imaging findings of liver tumours after percutaneous IRE ablation. From May 2016 to June 2019, 21 patients were treated with IRE at our institution. All patients met the following inclusion criteria: unresectable tumours; tumours unsuitable for thermal ablation because of close proximity to major veins; and biliary and venous systems of the liver that would cause heat-sink effects or collateral damage; these definitions were similar to the difficult location definitions set by Wei Yang et al. [13]. A history of hemihepatectomy was not considered a contraindication in the present study. Thermal ablation or catheter chemical ablation was not a contraindication. The exclusion criteria were a history of epilepsy or arrhythmia and the presence of an implanted cardiac pacemaker or metal biliary stent.

\section{IRE procedures}

All steps were performed under general anaesthesia using the NanoKnife IRE system (Angiodynamics Inc., Latham, NY, USA). A doctor with more than 5 years of experience in US-guided interventional procedures and IRE performed all IRE procedures. The IRE generator was programmed following the manufacturer's instructions. Based on the pre-IRE image, the NanoKnife System calculated related parameters, namely, the number of electrodes, the expected ablation area, the number of electrodes, and the distance between the electrodes. The ablation procedure is the same as that described in the previous study. The required number of needles was selected according to the nodule size. The applied voltage was $1800-3000 \mathrm{~V}$ (pulse length 70-90; pulse repetition number 90-270). Ablation was performed under ultrasound guidance.

\section{Imaging procedure}

US scanning was performed to assess the locations, sizes and margins of the tumours before ablation. The first image acquisition was conducted after the intervention (immediately after ablation), and follow-up imaging was performed to assess the development of the ablation zone.

CEUS was used to evaluate the effect of IRE. All CEUS studies were performed using Mylab 90 (Esaote, Italy) or ultrasound scanners equipped with $3.5-6-\mathrm{MHz}$ convex transducers. The US contrast agent used was SonoVue (Bracco SpA, Milan, Italy). After injecting $2.4 \mathrm{ml}$ of SonoVue agent, $5 \mathrm{~mL}$ of $0.9 \%$ saline was injected. Immediately after injection the ultrasound contrast agent, a dual-B mode image was acquired. Simultaneously, a timer was started. According to previous studies, we defined three phases: the arterial phase (15-30 s), the portal venous phase $(31-120 \mathrm{~s})$, and the late phase (121 s and later). The ablation zone was observed continuously for $5 \mathrm{~min}$. The entire image for inspection was recorded digitally and stored on the hard disk of the US scanner for subsequent analysis.

All CEUS studies were performed before ablation and immediately, 1 day, and 1 month after tumour ablation to evaluate the characteristics of the IRE ablation zone. MRI was conducted 1 month after ablation to investigate the therapeutic efficacy. 


\section{CEUS analysis}

One operator with 10 years of experience with CEUS performed all the CEUS examinations during the course of the study. Data were analysed and defined by consensus between two doctors. If different opinions arose, the reviewers jointly re-assessed the saved images and then reached a consensus. The presence or absence of tumour enhancement on the immediate CEUS image was recorded. The following imaging features were assessed: echogenicity, the boundary and the enhancement pattern of the IRE ablation zones.

\section{Statistical methods}

A p value of 0.05 was considered statistically significant. Descriptive statistics were used to present the results as absolute numbers (n), the means and standard deviations (SDs), or percentages. We compared the efficacy of CEUS assessment with MRI by Cohen's $\mathrm{K}$ values [14]. In addition, sensitivity, specificity, and positive and negative predictive values were all calculated. All statistical analyses were performed using SPSS software (version 23.0).

\section{Results}

The patient group included 21 patients (24 ablation lesions) aged $31-86$ years (mean $59.6 \pm 12.7$ years). The identity of the tumour could be histologically confirmed in 15 cases, while 9 lesions were diagnosed based on imaging. The histologic findings showed hepatocellular carcinoma (HCC) in 13 lesions and metastases of gastrointestinal tumours in 2 lesions.

\section{Immediate post-procedural assessment}

On conventional US performed immediately after IRE treatment, the ablated zones were either hypo-echoic $(17 / 24,71 \%)$ or iso-echoic $(7 / 24,29 \%)$. The boundaries were unclear, and the echogenicity of the boundary was heterogeneous. On CEUS, in 4 of the 24 ablated areas (17\%), no enhancement was observed, and the boundary was clearly outlined (Fig. 1a). Enhancement of the IRE ablation zone was observed in 20 of the 24 cases $(83 \%)$ : 1 ablation zone showed slight hyper-enhancement in the early arterial phase that was heterogeneous and washed out in the late arterial phase, and 19 ablation zones showed hypo-enhancement in the arterial phase. The boundaries between the enhanced and non-enhanced zones were clear.

\section{One-day follow-up}

B-mode imaging of the ablated zones performed 1 day after IRE treatment showed hyper-echoic foci $(13 / 24$, $54 \%)$, iso-echoic foci $(5 / 24,20 \%)$, hypo-echoic foci $(6 / 24$, $25 \%)$. The boundaries were clear. On CEUS, 16 of 24 ablated areas showed no enhancement at all (67\%) (Fig. 1b). Enhancement of the ablation zone was observed in 8 of
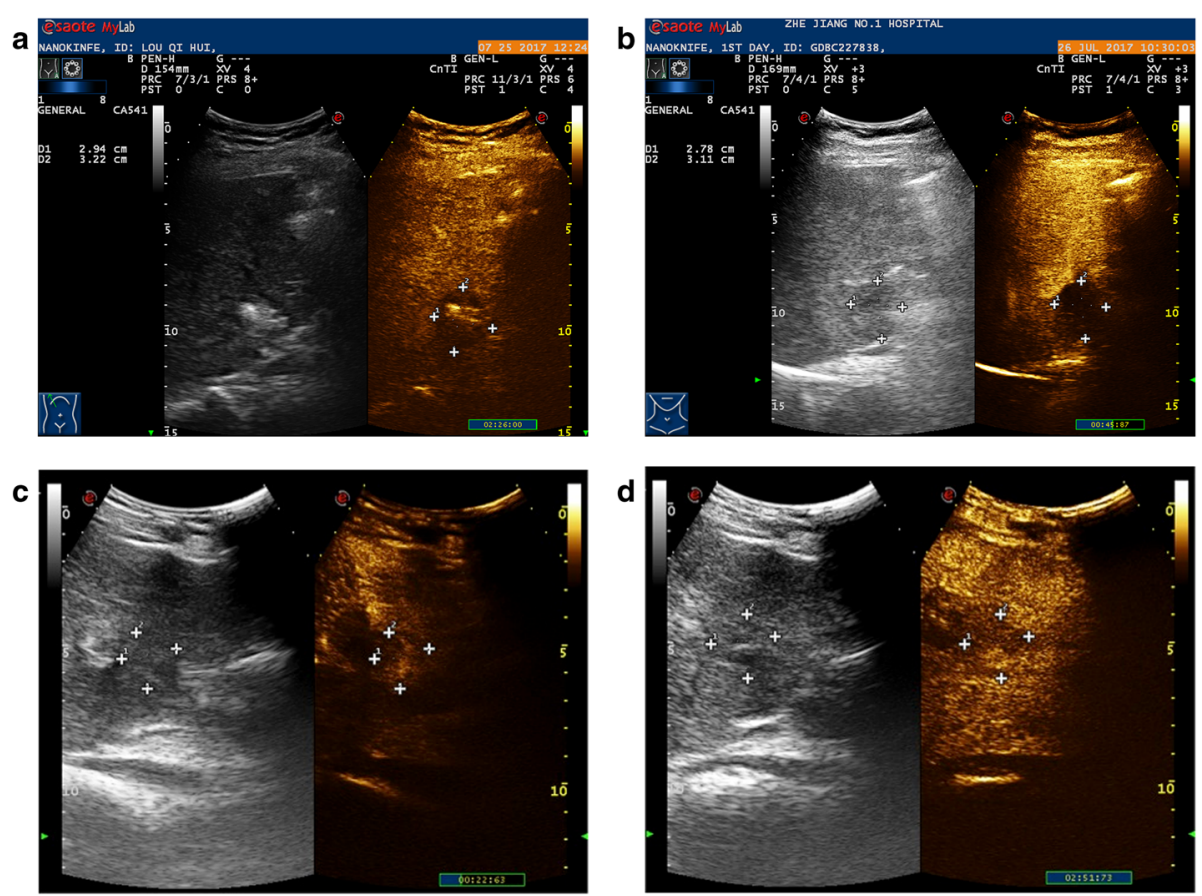

Fig. 1 a Immediate post-IRE CEUS demonstrated complete non-enhancement in the ablation zone. The boundary of the non-enhanced area was clearly outlined. b A follow-up scan one day after IRE also showed complete non-enhancement. c A 46-year-old man presented with hepatic carcinoma. One month after IRE ablation, a hyper-enhanced lesion was observed in the early arterial phase. $\mathbf{d}$ The hyper-enhanced foci washed out during the late arterial phase 
the 24 zones (33\%). One ablation zone showed slight hyper-enhancement in the early arterial phase, which washed out in the late arterial phase, while others showed hypo-enhancement in the arterial phase. During the portal venous and late phases, the enhanced foci showed hypo-enhancement.

\section{One-month and later follow-up examinations}

On conventional US performed 1 month after IRE treatment, 22 ablated zones showed hyper-echoic foci with clear boundaries (22/24, 92\%), and 2 ablated zones were still hypo-echoic $(2 / 21,10 \%)$. Complete non-enhancement was documented in 19 of the 24 ablation zones (79\%). One ablation zone showed slight hyper-enhancement in the early arterial phase that washed out in the late arterial phase (Fig. 1c and d). Another 4 zones showed quick wash-in and quick wash-out patterns on CEUS. No evidence of peripheral contrast enhancement could be found during the arterial phase or during the portal venous phase.

One month after IRE ablation, no evidence of recurrence was found by MRI in 17 ablation zones (17/24, $71 \%)$, while recurrence was identified in 7 zones $(7 / 24$, $29 \%)$. Substantial agreement $(\kappa=0.78 ; p<0.05)$ was observed between the results obtained with CEUS and those obtained with MRI. With MRI as the gold standard, the sensitivity, specificity, positive predictive value and negative predictive value of CEUS were $71.4 \%$, $100 \%, 100 \%$, and $89.5 \%$, respectively.

\section{Discussion}

IRE has attracted increasing interest because the nonthermal properties of this method [15] permit ablation of tumours adjacent to vital structures [16]. Previous studies have shown that the ultrasound findings of IRE applied to the liver will change from a few seconds to at least a few hours with time $[3,17,18]$. Our results show the evolution of post-IRE ablation patterns over a clinically relevant period.

As noted in an animal-based experiment, a strong association exists between conventional US and histopathology [5]. In our study, on conventional US, the IRE ablation zones of most patients appeared as a developing hypo-echoic area that demonstrated an increasingly hyperechogenic ablation zone starting one day after the procedure.

Our US findings after IRE ablation evolved sequentially over time. The findings showed good correlations with those in previous studies $[5,19]$. In the study, specimens obtained immediately after ablation showed that the enlarged oedematous sinusoidal space was mainly filled with fluid, and almost no haemorrhagic infiltration was observed. As time passed, the haemorrhagic infiltrate became more dominant, which might be due to the widened fluid-filled sinusoids at the beginning of the procedure. The authors attribute this hyper-echogenicity to red blood cell accumulation over time. The degree of erythrocyte infiltration was qualitatively related to echogenicity. Lee et al. also reported the status of 55 ablation zones immediately after ablation and 1 day after ablation and showed that the immediate low echogenicity of the treatment area was converted into total hyperechogenicity on the 1st postoperative day [19]. Appelbaum et al. speculated that red blood cells progressively infiltrated into deep regions of the ablated zone such that the hyper-echoic rim on US at 90-120 min transitioned to complete hyper-echogenicity within $24 \mathrm{~h}$ [5]. Concordance exists between our findings and those of previous studies describing the characteristics of images obtained after ablation.

In CEUS, most of the ablated zones showed hypoenhancement immediately after IRE. Chung et al. found the following zones with different enhancement patterns on CT perfusion images in normal porcine liver: an inner non-enhanced zone; a middle well-defined progressive internal enhancement zone, and an outer illdefined arterial enhancement zone. On histopathology, the inner and middle zones accounted for the extent of cell death [20]. The histological examination suggested that the apoptotic process was involved, with complete cell death caused by IRE according to pathophysiology.

The CEUS images of the IRE ablation zones in normal liver tissue differed from the images of liver tumours treated with IRE. Our study also found that 12 ablated zones showed hypo-enhancement immediately after IRE but became non-enhanced one day after IRE for various reasons. Lee et al. attributed the focal hyper-attenuation to the release of contrast medium into an ablation defect caused by an IRE-induced microvasculature leakage within the defect zone [21]. Because the zones became non-enhanced on the follow-up CEUS images, the hyper-enhancement was probably caused by extraluminal contrast material. Another opinion concerns differences in contrast agent concentrations. Guo et al. suggested that nanometre-scale pores in the tissue cell membrane caused contrast agent to accumulate in the IRE zone and allowed the contrast agent to be internalized into the intracellular environment rather than remaining extracellularly [22], which complicates evaluation of whether the viable tissue is a residual tumour after ablation. Therefore, follow-up CEUS is necessary to assess the viable portion. However, unlike normal regeneration activity, the residual tumour can continue growing and results in a newly enhanced region.

Most of the non-enhancement pattern on CEUS 1 month after ablation is a sign of effective IRE treatment, but false-negative results may occur. Such nonenhancement patterns are different to those of the thermal ablation zone after complete ablation. Previous 
histologic examinations of the ablation zones showed cell death caused by apoptosis [19]. Cell death is observed with full preservation of the peri-ablative zone structures, such as blood vessels and bile ducts.

In the present study, only one patient continuously showed an enhanced ablation zone immediately and on follow-up CEUS images. The ablation zone became hyper-enhanced during the early arterial phase, slightly washed out in the late arterial phase and appeared hypoenhanced in the late phase. To elucidate the reason for this pattern, the patient underwent liver biopsy one month after IRE. The physiology results showed small patchy necrosis, inflammatory fibrosis, tissue hyperplasia and foam cell aggregation. The result ruled out the possibility of recurrence. A reasonable explanation for this finding is hyperplasia of the inflammatory tissue. This result can cause confusion in clinical practice. Therefore, a sequential follow-up is essential. Further investigation is needed to study the histological and cytological mechanisms underlying this process.

In our study, only 2 ablation zones still appeared as hypo-echoic areas, and both zones were in the same patient. After 1 month of follow-up, recurrence occurred near one of the zones treated with IRE ablation. In a previous multi-institutional review from 2009 through 2012, 31\% of the patients had recurrence during a median follow-up of 18 months. Among the study population, $31 \%$ of the patients had recurrence, and $10.7 \%$ had local recurrences at the ablated site [4]. Previous studies have attributed such recurrences to electric field sinks resulting from the heterogeneous structure and conductivity of the liver [23]. Later work from the authors indicated that the IRE-treated extracellular matrix (ECM) provides an environment for activation and differentiation of progenitor cells [24], but the mechanism is not completely understood; in contrast, some studies have suggested that abnormal ECM affects cancer progression by directly promoting cellular transformation and metastasis as well as tumour-associated angiogenesis and inflammation, leading to the generation of a tumorigenic microenvironment [25]. The role of the IRE-spared tumour matrix in follow-up recurrences requires further research. Another theory is related to the size of the treated liver tumours. Niessen et al. found that large tumour volumes $\left(>5 \mathrm{~cm}^{3}\right)$ portended early local recurrence [26].

In our study, the intra-hepatic blood vessels and bile duct remained almost completely intact after IRE. The hepatohilar bile duct of only one patient showed an unclear contrast agent pattern immediately after IRE. After $15 \mathrm{~min}$, the phenomenon disappeared. However, a consensus on the mechanism of IRE has not yet been reached. This effect may be due to the high proportion of collagenous connective tissue. A further hypothesis is that gap junctions, which are present in large numbers in the muscularis propria of blood vessels and the bile duct walls, may act as a conductive structure for the electrical currents; thus, the current can pass from cell to cell without causing destruction of the cell membrane [14]. Whether the effects of IRE are caused by a thermal or non-thermal mechanism remains unclear.

This study has several limitations. First, our study had a small sample size over a span of more than 2 years. Second, 9 lesions of the tumour were not diagnosed histologically before IRE. The last limitation was that almost all of the patients included in our study group previously underwent right or left liver resection and thermal ablation or catheter chemical ablation. The origin of the ablation zones was heterogeneous. However, this circumstance mirrors the status of our clinical treatment strategies. IRE as a novel technique was not the first choice in our hospital. Only patients who experienced tumour recurrence after liver resection and thermal ablation were considered for IRE. Because of this situation, assessing the images of the ablated zones during subsequent follow-up imaging examinations is important. We conclude that CEUS may be a useful tool for assessing the characteristics of post-IRE ablation changes and is an effective method to evaluate the therapeutic efficacy 1 month after ablation. Further studies are needed to evaluate more patients to precisely depict the appearance of hepatic zones on CEUS.

\section{Abbreviations \\ IRE: Irreversible electroporation; CEUS: Contrast-enhanced ultrasound ; US: Ultrasound; MRI: Magnetic resonance imaging; RFA: Radiofrequency} ablation; MWA: Microwave ablation; ECM: Extracellular matrix

\section{Acknowledgements}

Not applicable.

\section{Authors' contributions}

Linyu Zhou participated in the study design and drafted the manuscript. Shanyu Yin and Weilu Chai analysed and interpreted the data. Qiyu Zhao and Guo Tian collected the clinical data and performed the follow-up. Linyu Zhou and Tianan Jiang participated in the study design, carried out the IRE ablation and provided final approval of the version to be published. All of the authors are in agreement with the manuscript's content.

\section{Funding}

This study was supported by National Natural Science Foundation of China (grant number: 81971623).

\section{Availability of data and materials}

The datasets used and/or analysed during the current study are available from the corresponding author upon reasonable request.

Ethics approval and consent to participate

This prospective study was approved by the ethics committee of the First Affiliated Hospital of Zhejiang University (Zhejiang, China). Informed consent for study participation was obtained.

Consent for publication

Not applicable. 


\section{Competing interests}

The authors declare that they have no competing interests.

\section{Author details}

'Department of Ultrasonography, The First Affiliated Hospital, College of Medicine, Zhejiang University, No. 79 Qingchun Road, Hangzhou 310003, Zhejiang Province, P.R. China. ${ }^{2}$ Department of Hepatobiliary and Pancreatic Surgery, The First Affiliated Hospital, College of Medicine, Zhejiang University, No. 79 Qingchun Road, Hangzhou 310003, Zhejiang Province, P.R. China. ${ }^{3}$ Collaborative Innovation Center for Diagnosis and Treatment of Infection Diseases, The First Affiliated Hospital, College of Medicine, Zhejiang University, Hangzhou, P.R. China.

Received: 19 June 2020 Accepted: 9 November 2020

Published online: 23 November 2020

\section{References}

1. Ferlay J, Colombet M, Soerjomataram I, Mathers C, Parkin DM, Pineros M, Znaor A, Bray F. Estimating the global cancer incidence and mortality in 2018: GLOBOCAN sources and methods. Int J Cancer. 2019;144:1941-53.

2. Weaver JC. Electroporation: a general phenomenon for manipulating cells and tissues. J Cell Biochem. 1993:51:426-35.

3. Lin MX, Kuang M, Xu M, Zhuang BW, Tian WS, Ye JY, Xie XH, Xie XY. Ultrasound and contrast-enhanced ultrasound for evaluation of irreversible electroporation ablation: In Vivo proof of concept in normal porcine liver. Ultrasound Med Biol. 2016a:42:2639-49.

4. Philips P, Hays D, Martin RC. Irreversible electroporation ablation (IRE) of unresectable soft tissue tumors: learning curve evaluation in the first 150 patients treated. PLoS One. 2013;8:e76260.

5. Appelbaum L, Ben-David E, Sosna J, Nissenbaum Y, Goldberg SN. US findings after irreversible electroporation ablation: radiologic-pathologic correlation. Radiology. 2012;262:117-25.

6. Guibal A, Bertin C, Egels S, Savier E, Grenier PA, Lucidarme O. Contrastenhanced ultrasound (CEUS) follow-up after radiofrequency ablation or cryoablation of focal liver lesions: treated-area patterns and their changes over time. Eur Radiol. 2013;23:1392-400.

7. Figini M, Wang X, Lyu T, Su Z, Procissi D, Yaghmai V, Larson A, Zhang Z. Preclinical and clinical evaluation of the liver tumor irreversible electroporation by magnetic resonance imaging. Am J Trans Res. 2017;9:580-90. .

8. Hu S, Sun C, Wang B, Zhou K, Pan L, Shangguan J, Yang J, Yaghmai V, Figini $M$, Zhang Z. Diffusion-Weighted MR Imaging to Evaluate Immediate Response to Irreversible Electroporation in a Rabbit VX2 Liver Tumor Model. J Vasc Interventional Radiol: JVIR. 2019;30:1863-9. .

9. Lee Y, Lu D, Osuagwu F, Lassman C. Irreversible electroporation in porcine liver: short- and long-term effect on the hepatic veins and adjacent tissue by $C T$ with pathological correlation. Invest Radiol. 2012;47:671-5.

10. Lin M, Kuang M, Xu M, Zhuang B, Tian W, Ye J, Xie X, Xie X. Ultrasound and contrast-enhanced ultrasound for evaluation of irreversible electroporation ablation: In Vivo proof of concept in normal porcine liver. Ultrasound Med Biol. 2016b;42:2639-49. .

11. Rennert J, Wiesinger I, Schicho A, Wiggermann P, Stroszczynski C, Beyer L, Jung E. Color Coded Perfusion Imaging with Contrast Enhanced Ultrasound (CEUS) for Post-Interventional Success Control Following Irreversible Electroporation (IRE) of Primary and Secondary Malignant Liver Lesions. J Gastrointest Liver Dis: JGLD. 2019;28:311-8.

12. Niessen C, Beyer L, Haimerl M, Schicho A, Stroszczynski C, Wiggermann P, Jung E. Percutaneous irreversible electroporation of hepatocellular carcinoma: Contrast-enhanced ultrasound-findings during 1-year follow-up ClinHemorheolMicrocirc. 2019:72:85-93.

13. Yang W, Yan K, Wu GX, Wu W, Fu Y, Lee JC, Zhang ZY, Wang S, Chen MH Radiofrequency ablation of hepatocellular carcinoma in difficult locations: Strategies and long-term outcomes. World J Gastroenterol. 2015;21:1554-66.

14. Lencioni R, Llovet JM. Modified RECIST (mRECIST) assessment for hepatocellular carcinoma. Semin Liver Dis. 2010;30:52-60. .

15. Faroja M, Ahmed M, Appelbaum L, Ben-David E, Moussa M, Sosna J, Nissenbaum I, Goldberg SN. Irreversible electroporation ablation: is all the damage nonthermal? Radiology. 2013;266:462-70.

16. Lee EW, Thai S, Kee ST. Irreversible electroporation: a novel image-guided cancer therapy. Gut Liver. 2010;4(Suppl 1):S99-104.

17. Granata V, de Lutio di Castelguidone E, Fusco R, Catalano O, Piccirillo M, Palaia R, Izzo F, Gallipoli AD, Petrillo A. Irreversible electroporation of hepatocellular carcinoma: preliminary report on the diagnostic accuracy of magnetic resonance, computer tomography, and contrast-enhanced ultrasound in evaluation of the ablated area. Radiol Med. 2016;121:122-31 .

18. Wiggermann P, Zeman F, Niessen C, Agha A, Trabold B, Stroszczynski C, Jung EM. Percutaneous irreversible electroporation (IRE) of hepatic malignant tumours: contrast-enhanced ultrasound (CEUS) findings. Clin Hemorheol Microcirc. 2012;52:417-27.

19. Lee EW, Chen C, Prieto VE, Dry SM, Loh CT, Kee ST. Advanced hepatic ablation technique for creating complete cell death: irreversible electroporation. Radiology. 2010;255:426-33. .

20. Chung DJ, Sung K, Osuagwu FC, Wu HH, Lassman C, Lu DS. Contrast enhancement patterns after irreversible electroporation: experimental study of CT perfusion correlated to histopathology in normal porcine liver. J VasclntervRadiol. 2016;27:104-11.

21. Lee YJ, Lu DS, Osuagwu F, Lassman C. Irreversible electroporation in porcine liver: acute computed tomography appearance of ablation zone with histopathologic correlation. J Comput Assist Tomogr. 2013;37:154-8. .

22. Guo Y, Zhang Y, Nijm GM, Sahakian AV, Yang GY, Omary RA, Larson AC. Irreversible electroporation in the liver: contrast-enhanced inversionrecovery MR imaging approaches to differentiate reversibly electroporated penumbra from irreversibly electroporated ablation zones. Radiology. 2011; 258:461-8.

23. Golberg A, Yarmush ML. Nonthermal irreversible electroporation: fundamentals, applications, and challenges. IEEE Trans Biomed Eng. 2013;60: 707-14.

24. Golberg A, Bruinsma BG, Jaramillo M, Yarmush ML, Uygun BE. Rat liver regeneration following ablation with irreversible electroporation. PeerJ. 2016:4:e1571.

25. Lu P, Weaver VM, Werb Z. The extracellular matrix: a dynamic niche in cancer progression. J Cell Biol. 2012;196:395-406. .

26. Niessen C, Igl J, Pregler B, Beyer L, Noeva E, Dollinger M, Schreyer AG, Jung EM, Stroszczynski C, Wiggermann P. Factors Associated with Short-Term Local Recurrence of Liver Cancer after Percutaneous Ablation Using Irreversible Electroporation: A Prospective Single-Center Study. J Vasc Interv Radiol. 2015;26:694-702.

\section{Publisher's Note}

Springer Nature remains neutral with regard to jurisdictional claims in published maps and institutional affiliations.
Ready to submit your research? Choose BMC and benefit from:
- fast, convenient online submission
- thorough peer review by experienced researchers in your field
- rapid publication on acceptance
- support for research data, including large and complex data types
- gold Open Access which fosters wider collaboration and increased citations
- maximum visibility for your research: over $100 \mathrm{M}$ website views per year
At BMC, research is always in progress.
Learn more biomedcentral.com/submissions 